\title{
Colon Cancer Screening Strategies to improve prevention and early detection
}

\author{
Luiz Ronaldo Alberti* \\ Associate Professor of the Department of Surgery, Federal University of Minas Gerais, and Santa Casa de Belo Horizonte, Brazil
}

Submission: February 06, 2017; Published: February 23, 2017

"Corresponding author: Luiz Ronaldo Alberti, Department of Surgery, Federal University of Minas Gerais, Avenida Alfredo Balena, 190. Centro, Belo Horizonte, Minas Gerais, Brazil, Tel: +55-31-34099759; Fax: +55-31-34099759, Email: luizronaldoa@yahoo.com.br

\section{Opinion}

Colorectal carcinoma (CRC) is a common cause of death and may be prevented by routine screening strategies in order to detect precancerous lesions and early cancers [1]. However, colorectal screening is under used, and at least $40 \%$ of ageeligible adults do not adhere to up-to-date screening guidelines. Three strategies may increase compliance on colon cancer screening rates: to convince the population and medical doctors about the importance of undergoing a screening test; to achieve higher efficacy in standard screening tests; to develop new more sensitive and efficacious screening methods.

Physicians are increasingly aware of the importance of screening to reduce mortality caused by CRC. Despite this fact, many patients do not receive this needed recommendation. This recommendation is of vital importance in order to convince patients to prevent CCR [2].

When screening tests are considered, the perfect knowledge of each exam limits and periodicity is necessary to make the CRC screening test more efficacious. Colonoscopy is an important method and is largely used to evaluate the colon. As a major advantage, this test frequently allows for the treatment of some affections immediately upon diagnosis (e.g. polypectomy, dilatation, hemostasis), behaving as a propedeutic and therapeutic method [3].

When properly executed by a well-trained professional, under adequade bowel preparation, a colonoscopy can be considered safe, precise, and easily tolerated by patients. American Society for Gastrointestinal Endoscopy (ASGE) and the American College of Gastroenterology (ACG) Task Force on Quality in Endoscopy defined quality indicator in colonoscopy to improve the quality of the exam and reduce complications, especially the number of missed [4]. These indicators were organized in three moments: before, during, and after the procedure. Every endoscopist must understand and target each item. Knowing the technique is not enough; it must also be well-executed. The main value of a colonoscopy as a screening method depends on the quality of the exam, as the findings (particularly polyps) are definitive to determining the interval of future colonoscopies. The most important quality indicators are: Quality of bowel preparation, Ceccum intubation, Adenoma detection rate (ADR) in asymptomatic average risk patients: must be over $25 \%$ in screened populations and Withdrawal tim [4]. Ideally, all endoscopists should measure, register, and interpret their own quality indicators in colonoscopy. Colonoscopy, to be costeffective as a CRC screening method, must be executed according to quality indicator parameters [4].

The patient should be recommended to an adequate pstcolonoscopy follow-up. Colonoscopy intervals are a key-points in CRC screening. This interval is often a decision made by the physician who requested the first exam. However, not all nonendoscopists know how to correctly interpret the results of colonoscopy exams and hystological findings to determine the best interval. In these situations, there is a tendency to shorten intervals. Unnecessary and early request of colonoscopy commits its cost-effectiveness, exposes patients to unnecessary risks, and onerates the health care system.

The most recent recommendation regarding postpolypectomy surveillance was published in 2012 [5] and adapted as a clinical decision tool from AGA in 2014. They recommend follow-up based on endoscopic and hystological findings. To follow the recommendations, a complete exam (up to the ceccum) must be performed, with excellent quality of bowel preparation and the complete removal of all polyps. If any of these criteria are not attained, future exam intervals must be reduced [5]. The need to detect colorectal adenomas and cancer has led to the implementation of new methods and technology and in upon current colonoscopy technology. 
Stool DNA testing (Fecal DNA testing - COLOGUARD) is a stool-based test intended for the qualitative detection of colorectal neoplasia associated with DNA markers and with the presence of occult hemoglobin in human stool samples. This method is available and approved by the US FDA in 2014 [6].

Check cap is a capsule device that produces images of the colon using low dose radiation and creates a 3-dimensional reconstructed image of the colon surface. Capsule is swallowed by the patient and no bowel preparation is needed. This method is under investigation [7].

Colon capsule endoscopy (CCE) is awireless, minimally invasive technique for the imaging of the large bowel. It also may be preferable for high risk patients or who have had problems such as incomplete exams in the past. Their disadvantages are: high costs, the need for bowel cleansing and inability to take biopsies. [8].Current indications target patients on whom conventional colonoscopy cannot be or has been incompletely performed [29]. Other potential applications, such as CCR screening and surveillance of inflammatory bowel disease still require further clarification [8].

Technological advances in colonoscopy intend to improve visualization of the proximal aspects of colonic folds, anatomic flexures in order to avoid missed lesions. In this way, Third Eye ${ }^{\circledR}$ technology (Retroscope and Panoramic) is an auxillary, throughthe-scope device able to retroflex $180^{\circ}$ and is intended to visualize proximal folds and at the anatomical flexures of the colon. A video camera and a light-emitting diode (LED) illumination is located in the tip of the device, providing continuous retrograde image during the procedure [9].

Other devices provides a high resolution, with higher field of view such as Fuse ${ }^{\circledR}$ Full Spectrum Endoscopy ${ }^{\circledR}$ colonoscopy platform and Extra-Wide-Angle-View colonoscope [10]. The NaviAid $^{\text {TM }}$ G-EYE ${ }^{\text {TM }}$ Systemis is a colonoscope with a balloon, which can be inflated, attached to the flexible tip of a standard colonoscope [11]. The mechanical flattening and straightening of haustral folds with the inflated balloon allows one to view hidden anatomical areas, thus increasing adenoma detection. Reported that the NaviAid ${ }^{\text {TM }}$ G-EYE ${ }^{\text {TM }}$ balloon colonoscopy detected $81 \%$ more adenomas $(\mathrm{P}<0.001)$ than did the standard colonoscope.
Procedure time and the incidence of complete colonoscopy with cecal intubation did not differ between groups. No adverse events were reported $[11,12]$.

\section{References}

1. James AS, Richardson V, Wang JS, Proctor EK, Colditz GA (2013) Systems intervention to promote colon cancer screening in safety net settings: protocol for a community-based participatory randomized controlled trial. Implement Sci 8: 58.

2. Sifri R, Wender R, Lieberman D, Potter M, Peterson K, et al. (2010) Developing a quality screening colonoscopy referral system in primary care practice: a report from the national colorectal cancer roundtable. CA Cancer J Clin 60(1): 40-49.

3. Burt RW (2000) Colon cancer screening. Gastroenterology 119(3): 837-853.

4. Hawes RH, Lowry A, Deziel D (2006) A consensus document on bowel preparation before colonoscopy: Prepared by a Task Force From The American Society of Colon and Rectal Surgeons (ASCRS), The American Society for Gastrointestinal Endoscopy (ASGE), and The Society for Gastrointestinal and Endoscopic Surgeons (SAGES). Gastrointest Endosc 63(7): 894-909.

5. Lieberman DA, Rex DK, Winawer SJ, Giardiello FM, Johnson DA, et al. (2012) Guidelines for colonoscopy surveillance after screening and polypectomy: a consensus update by yhe US Multi-Society Task Force on colorectal cancer. Gastroenterology 143(3): 844.

6. Rex DK, Schoenfeld PS, Cohen J, Pike I, Adler DG, et al. (2014) Quality indicators for colonoscopy. Gastrointest Endosc 81(1): 31-53.

7. Imperiale $\mathrm{TF}$, Ransohoff DF, Itzkowitz SH, Levin TR, Lavin $\mathrm{P}$, et al. (2014) Multitarget Stool DNA Testing for Colorectal-Cancer Screening. N Engl J Med 370: 1287-1297.

8. Check-Cap - The first non-invasive, prep-free imaging test for colon cancer.

9. Spada C, Barbaro F, Andrisani G, Minelli Grazioli L, Hassan C, et al. (2014) Colon capsule endoscopy: What we know and what we would like to know. World J Gastroenterol 20(45): 16948-16955.

10. Gralnek IM (2015) Emerging technological advancements in colonoscopy: Third Eye $₫$ Retroscope $₫$ and Third Eye $₫$ Panoramic TM, Fuse ${ }^{\circledR}$ Full Spectrum Endoscopy ${ }^{\circledR}$ colonoscopy platform, ExtraWide-Angle-View colonoscope, and NaviAid TMG-EYETM balloon colonoscope. Digestive Endoscopy 27(2): 223-231.

11. Uraoka T, Tanaka S, Matsumoto T, Matsuda T, Oka S, et al. (2013) A novel extra-wideangle-view colonoscope: A simulated pilot study using anatomic colorectal models. Gastrointest. Endosc 77(3): 480-483.

12. Shpak B, Halpern Z, Kiesslich R (2013) A novel balloon colonoscope for increased polyp detection rate - results of a randomized tandem study. UEGW 2013 (abstract \#OP286).
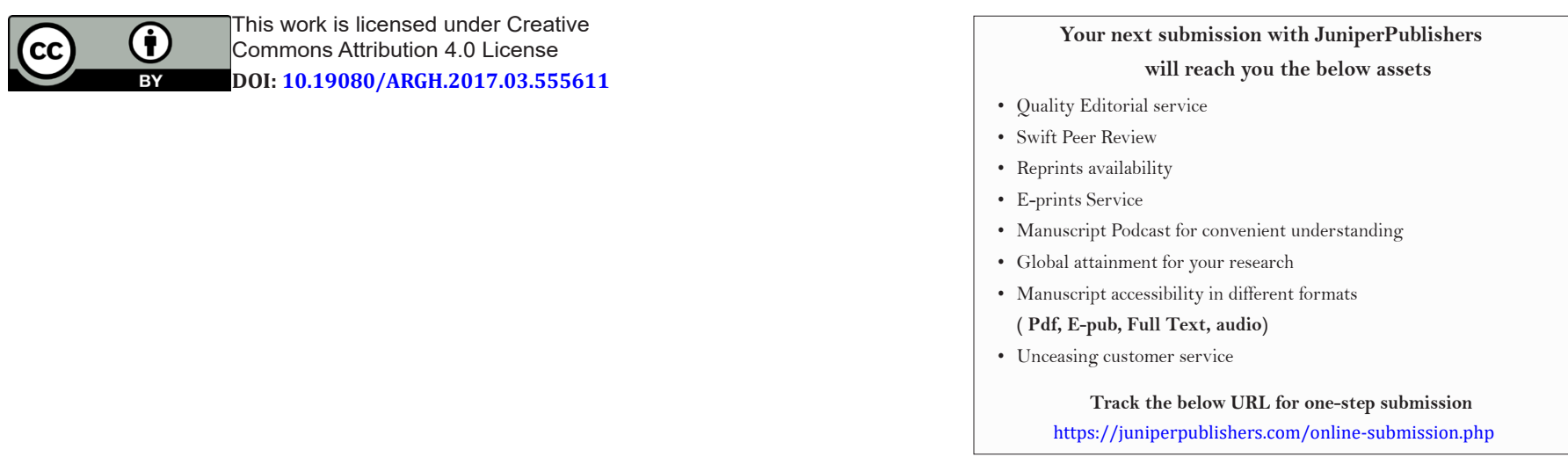